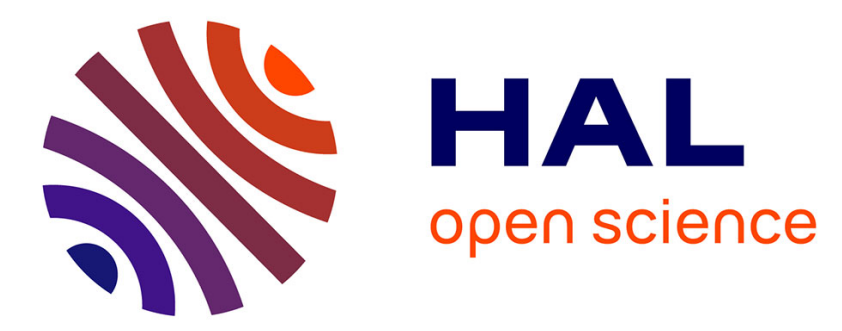

\title{
Dynamic Analysis of Fretting-Wear in Friction Contact Interfaces
}

Loic Salles, Laurent Blanc, Fabrice Thouverez, Alexander M Gouskov

\section{To cite this version:}

Loic Salles, Laurent Blanc, Fabrice Thouverez, Alexander M Gouskov. Dynamic Analysis of FrettingWear in Friction Contact Interfaces. Journal of Engineering for Gas Turbines and Power, 2010, 132 (1), 10.1115/1.3028229 . hal-02529258

\section{HAL Id: hal-02529258 \\ https://hal.science/hal-02529258}

Submitted on 2 Apr 2020

HAL is a multi-disciplinary open access archive for the deposit and dissemination of scientific research documents, whether they are published or not. The documents may come from teaching and research institutions in France or abroad, or from public or private research centers.
L'archive ouverte pluridisciplinaire HAL, est destinée au dépôt et à la diffusion de documents scientifiques de niveau recherche, publiés ou non, émanant des établissements d'enseignement et de recherche français ou étrangers, des laboratoires publics ou privés. 


\title{
Dynamic Analysis of Fretting-Wear in Friction Contact Interfaces
}

\author{
Loïc Salles, ${ }^{1,2,3, *}$ Laurent Blanc, ${ }^{1}$ Fabrice Thouverez, ${ }^{1}$ and Alexander M. Gouskov ${ }^{3}$ \\ ${ }^{1}$ École Centrale de Lyon, Laboratoire de Tribologie et Dynamique des Systèmes \\ 36 avenue Guy de Collongue, 69134 Ecully Cedex, France \\ ${ }^{2}$ Snecma - Safran group \\ 77550 Moissy-Cramayel, France \\ ${ }^{3}$ Moscow State University of Technology named after Bauman, Moscow, Russia
}

(Dated: May 6, 2008)

\begin{abstract}
Fretting wear is a very important phenomenon occurring in bladed disks. It causes that blades must be replaced in turbomachines during their life-cycle. Methods exist to predict fretting-wear in quasi-static analysis. However they don't predict all the phenomena observed in blade attachments on real turbomachines. That's why this study assumes that dynamics plays a role in fretting-wear. This paper is devoted to the realistic modelling and calculation of fretting-wear and dynamical response of structures in unilateral contact with friction. Vibration and wear phenomena present very different scales both in time and space. Therefore the difficulty is in finding methods that enable one to solve the non-linear problem with a good compromise between the approximations made about the dynamical aspects and those linked with fretting-wear issues. Here, phenomenological examples are studied. They involve a small number of degrees of freedom with a view to understanding the complex coupling between vibration and fretting-wear. This way, they will show the relative importance of parameters.
\end{abstract}




\section{NOMENCLATURE}

$F_{c} \quad$ Nodal force of contact.

$K_{w} \quad$ Wear coefficient in Archard's law.

M, C, K Mass, viscous damping and stiffness matrices.

$\boldsymbol{U} \quad$ Time-domain displacement vector.

W Wear depth.

$\mathbf{Z}_{r} \quad$ Reduced dynamic stiffness.

- $\quad$ Multiharmonic vectors

$-r \quad$ Relative value

$-_{-}^{T},{ }_{-}^{N} \quad$ Tangential and normal direction

$\eta \quad$ Slow scale of time.

$\boldsymbol{\lambda}, \tilde{\boldsymbol{\lambda}}$ Lagrangian multiplier in time and frequency domains.

\section{INTRODUCTION}

One of the most important sources of nonlinearities in turbine is due to contact with friction at the interfaces. These nonlinearities often have a positive aspect because they introduce damping in the vibrational response. Especially, designers can put friction dampers as underplatform damper, ring damper, impact damper,... to reduce the amplitude of resonances. But relative displacements at the interface have a damaging aspect, since frettingwear can appear and induce cracking. Recent studies ${ }^{12}$ permit to calculate the vibrational response of bladed disks in the presence of friction in blade attachments. They can predict the dissipated energy at the interfaces. On the other hand, most fretting-wear studies in blade roots are performed in quasi-static situations. Thus, in the case of planes, they only calculate $^{3}$ or test ${ }^{4}$ the take-off or landing situations. Nevertheless, dynamic wear can also occur when vibrations appear at cruising rate. In quasi-static situations, displacements are more important compared with dynamic situations. But in this latter case, in spite of the fact that only micro-slidings are observed, they are repeated during a much longer time. Current numerical modelling doesn't match with the real wear observed in turbomachinery. That is why this study assumes that dynamics plays a role in fretting-wear. Because of the complexity it introduces, this hypothesis is very seldom taken into account in literature ${ }^{5,6}$.

Wear is a very complex phenomenon because hardness, plasticity, grain structure, tem- 
perature,... can change wear debris creation. According to ${ }^{7}$ around 180 laws of wear have been proposed. Recently, a new model based on dissipated energy has been proposed to quantify wear directly ${ }^{8}$. To quantify wear, the Archard's model ${ }^{9}$ is the most commonly used. It considers the wear volume is linked to the product of the normal force and of the sliding displacement. Classically, wear coefficient quantification is then performed through the representation of the evolution of the worn volume as a function of the normal load. The loss of matter can be known through the measurement of the loss of mass, of the loss of dimensions, of the evolution of Vickers microhardness imprints or directly by surface profilometry. This model will be used in this paper. Hardness will be considered constant. The plasticity of the material will be neglected.

Different numerical approaches exist to compute wear on structures. The most common one is the Finite Element Method (FEM) ${ }^{10,11}$. This tool is efficient to compute wear but it is necessary to create a very fine meshing to calculate contact normal forces. Because mesh updating is necessary to take wear into account, calculations are time consuming. A smart and more economical method is based on Winkler's foundation to predict wear ${ }^{12}$. Semi-analytical or Boundary Element methods have been also developed to predict wear ${ }^{3,13}$. Gallego's method is an acceleration technique which efficiently improves the convergence rate. But these methods are difficult to use when the problem of contact with friction takes dynamics effects into account. In this paper we will introduce an approach based on the FEM to predict wear in dynamics. The main assumption is that a periodic steady state is reached and that wear will modify this state a little. Thus, it is possible to use methods based on Multi Harmonic Balance and Alternate Frequency Time procedures ${ }^{14-16}$.

Here, is investigated the dynamical behaviour of two structures in contact with friction and wear. To start with, a simple system with two degrees of freedom is treated numerically. It will enable one to understand the phenomena coupling wear and dynamics. In case of total slip, it would be possible, to a certain extent, to uncouple the dynamic analysis and the fretting wear calculation. In the family of cases treated here, wear provokes the relaxation of the normal pressure, inducing strong coupling. On bladed disks, this phenomenon occurs especially under partial slip conditions, as shown in ${ }^{17}$. The final purpose will be to apply the method introduced here to a more realistic bladed disk. 


\section{METHOD OF ANALYSIS}

\section{A. Equations of Motion and Wear - Variational Formulation}

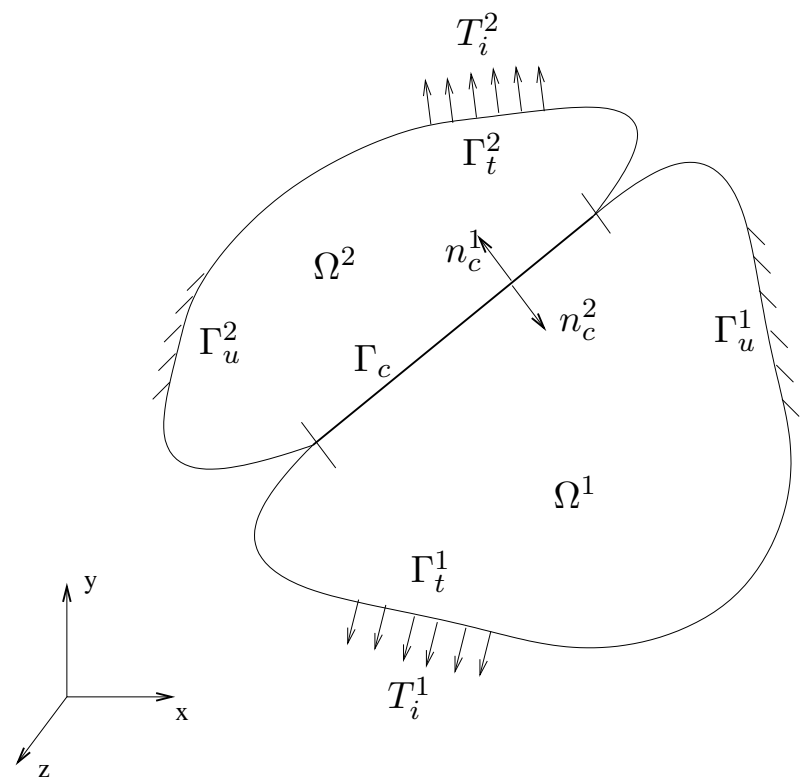

Figure 1: Description of the problem of contact between two solids

Let us consider the case of two elastic solids in contact with friction figure 1 . Both solids occupy two distinct domains $\Omega^{l}$ with smooth boundaries $\partial \Omega^{l}$. The boundary $\partial \Omega^{l}$ is divided into three disjoint parts $\Gamma_{t}^{l}, \Gamma_{u}^{l}$ and $\Gamma_{c}$. Traction forces are imposed on the first part of the boundary, displacements on the second and contact conditions on the third part of the surface. This part of body is unilaterally constrained in the direction normal to the surface and the Coulomb's law constrains the tangential directions. Moreover the wear phenomena occur on this part of the body. Following the formalism described by Stromberg ${ }^{18}$, let us build the constitutive equations for an elastic body with a contact interface in situation of fretting-wear. This formalism introduces a free energy and a pseudo-potential which correspond to Signorini unilateral contact conditions, Coulomb's law of friction and Archard's law of wear (4). The details about the way to obtain these free energy and pseudo-potential can be found in Stromberg ${ }^{18}$ and Klarbring ${ }^{19}$. It originates from the works of Lemaitre and Chaboche ${ }^{20}$. In this paper we will only present the variational formulation associated with the dynamical fretting-wear problem. 
For each domain $\Omega^{l}$, on a time interval $[0, T]$, find $u:[0, T] \rightarrow \mathcal{V}, \mathcal{W}=p_{N}:[0, T] \rightarrow \mathcal{K}_{N}$ and $p_{T}:[0, T] \rightarrow \mathcal{F}\left(P_{N}\right)$ such that for each time $t \in[0, T]:$

$$
\begin{gathered}
\int_{\Omega} \rho \ddot{u}_{i} \dot{v}_{i} d V+\int_{\Omega} E_{i j k l} \frac{\partial u_{k}}{\partial x_{l}} \frac{\partial v_{i}}{\partial x_{j}} d V+\int_{\Gamma_{c}} p_{i} v_{i} d A-\int_{\Gamma_{t}} t_{i} v_{i} d A=0 \quad \forall \mathbf{v} \in \mathcal{V}, \\
\int_{\Gamma_{c}}\left(u_{N}-w-g\right)\left(p_{N}^{\prime}-p_{N}\right) d A \leq 0 \quad \forall p_{N}^{\prime} \in \mathcal{K}_{N}, \\
\int_{\Gamma_{c}} \dot{u}_{T_{\alpha}}+\left(p_{T_{\alpha}}^{\prime}-p_{T_{\alpha}}\right)+\dot{w}\left(\mathcal{W}^{\prime}-\mathcal{W}\right) d A \leq 0 \quad \forall\left(p_{T}^{\prime}, \mathcal{W}^{\prime}\right) \in \mathcal{F}\left(p_{N}\right), \\
\dot{w}=k_{w}\left|p_{N}\right|\left\|\dot{\mathbf{u}}_{T}\right\|,
\end{gathered}
$$

where,

$$
\begin{aligned}
\mathcal{V} & =\left\{\mathbf{v} \mid \mathbf{v}(\mathbf{x})=0, \mathbf{x} \in \Gamma_{u}\right\} \\
\mathcal{K}_{N} & =\left\{p_{N} \mid p_{N}(\mathbf{x}) \geq 0, \mathbf{x} \in \Gamma_{c}\right\} \\
\mathcal{F}\left(p_{N}\right) & =\left\{\left(\mathbf{p}_{T}, \mathcal{W}\right) \mid\left(\mathbf{p}_{T}(\mathbf{x}), \mathcal{W}(\mathbf{x})\right) \in F\left(p_{N}\right), \mathbf{x} \in \Gamma_{c}\right\}
\end{aligned}
$$

These equations are respectively the principle of virtual work, the equivalent formulation of the Signorini's unilateral contact conditions, the inequality defining the complementary law of Coulomb's and the law of wear. $[0, T]$ represents the studied period, $\mathcal{W}$ is the dual of $w, F\left(p_{N}\right)$ is a closed convex set describing the friction and the wear limit criterion such that $F\left(p_{N}\right)=\left\{\left\|\boldsymbol{p}_{T}\right\|-\mu\left|p_{N}\right|+k_{w} p_{N} \mathcal{W}-k_{w} p_{N}^{2} \leq 0\right\}$ and $k_{w}$ coefficient of wear in Archard's law. ${ }_{K} w$ must be determinated experimentally. Mccoll ${ }^{10}$ determines an averaged wear coefficient from the measured wear profile for a cylinder/flat contact. He integrates this averaged coefficient in discretized Archard's law and simulates wear evolution in a"wear box"(FEM). In our case evaluation of averaged wear coefficient can be performed for a punch plan contact. This coefficient need to be discrtized to the nodes in contact in finite element discretization.

\section{B. Finite Element Discretization}

The numerical discretization of (1) is based on the FEM. In the case of a dynamical study, a component mode synthesis can be coupled with the FEM. However, all the degrees of freedom where friction and wear takes place must then be retained as physical coordinates. When both structures $(l=1,2)$ are in contact, we can write the equations of motion for 
each structure as:

$$
\mathbf{M} \ddot{\boldsymbol{U}}^{(l)}+\mathbf{C} \dot{\boldsymbol{U}}^{(l)}+\mathbf{K} \boldsymbol{U}^{(l)}+\boldsymbol{F}_{\boldsymbol{c}}^{(l)}\left(\boldsymbol{U}^{(l)}, \dot{\boldsymbol{U}}^{(l)}, W^{(l)}\right)=\boldsymbol{F}_{\boldsymbol{e x}}^{(l)},
$$

$\boldsymbol{F}_{\boldsymbol{e x}}$ is the vector of external forces (periodic excitation at frequency $\omega$ ) and $\boldsymbol{F}_{\boldsymbol{c}}$ represents the non-linear contact forces due to friction and impact. They also depend on the wear and on the materials in contact. The equation of wear (Archard's law), which is continuous, is also discretized by FEM. Wear is calculated at the nodes (marked $M$ ) of the interface:

$$
\dot{\boldsymbol{w}}^{M}=\frac{k_{w}}{I^{M}}\left|P_{N}^{M}\right|\left\|\dot{\boldsymbol{U}}_{T}^{M}\right\|
$$

$I^{M}$ are the weighting factors for each node $M, P_{N}$ is the nodal normal force and $\dot{\boldsymbol{U}}_{T}^{M}$ is the tangential velocity of the node $M$. The constraints introduced by Signorini's contact conditions and Coulomb's friction are, in the FEM formalism:

$$
\begin{aligned}
{ }^{t}\left(\boldsymbol{U}_{N}-\boldsymbol{W}-\boldsymbol{G}\right)\left(\boldsymbol{P}_{N}^{\prime}-\boldsymbol{P}_{N}\right) \leq 0 & \forall \boldsymbol{P}_{N}^{\prime} \in \mathcal{K}_{N}^{h} \\
{ }^{t} \dot{\boldsymbol{U}}_{T}\left(\boldsymbol{P}_{T}^{\prime}-\boldsymbol{P}_{T}\right) \leq 0 & \forall \boldsymbol{P}_{T}^{\prime} \in \mathcal{F}^{h}\left(\boldsymbol{P}_{N}\right),
\end{aligned}
$$

where $\mathcal{K}_{N}^{h}=\left\{P_{N}^{M}: P_{N}^{M} \geq 0\right\}$ is the approximation of $\mathcal{K}_{N}$ and $\mathcal{F}^{h}\left(\boldsymbol{P}_{N}\right)=$ $\left\{\boldsymbol{P}_{T}^{M}:\left\|\boldsymbol{P}_{T}^{M}\right\|-\mu\left|\boldsymbol{P}_{N}^{M}\right| \leq 0\right\}$ is the approximation of $\mathcal{F}\left(p_{N}\right)$ with $\mathcal{W}^{M}=\boldsymbol{P}_{N}^{M}$.

\section{Frequency domain formulation}

Without wear

When wear is neglected the steady-state motion is assumed to be periodic. We introduce Fourier series expansions to express displacements and forces as multi-harmonic vectors.

$$
\boldsymbol{U}(t)=\tilde{\boldsymbol{U}}_{0}+\sum_{n=1}^{N h} \tilde{\boldsymbol{U}}_{n, c} \cos (n \omega t)+\tilde{\boldsymbol{U}}_{n, s} \sin (n \omega t)
$$

and the multiharmonic vector is

$$
\tilde{\boldsymbol{U}}=\left[\tilde{\boldsymbol{U}}_{0}, \tilde{\boldsymbol{U}}_{1, c}, \tilde{\boldsymbol{U}}_{1, s}, \cdots, \tilde{\boldsymbol{U}}_{N_{h}, s}\right]^{T}
$$

A Galerkin procedure is performed to formulate the equations (5) in the frequency domain. The size of the problem can be reduced through two exact reductions in the frequency domain on the degrees of freedom involved in the contact elements and on the relative degrees of 
freedom. This procedure is detailed by Nacivet et al. ${ }^{2}$. The equations of motion take then the following form:

$$
\mathrm{Z}_{\mathbf{r}} \tilde{U}_{\boldsymbol{r}}+\tilde{\boldsymbol{\lambda}}=\tilde{\boldsymbol{F}}_{\boldsymbol{r}}
$$

where $\tilde{\boldsymbol{U}}_{\boldsymbol{r}}$ and $\tilde{\boldsymbol{F}}_{\boldsymbol{r}}$ are the multiharmonic vectors which represent the relative contact displacements and the reduced external forces. $\mathbf{Z}_{\mathbf{r}}$ is the reduced dynamic stiffness. The second reduction is possible thanks to the assumption of little displacements. In the node-to-node formulation, the Lagrange multipliers $\tilde{\lambda}$ are equal to the unknown contact forces. A nonlinear solver is used to find the zeros of the function:

$$
f\left(\tilde{\boldsymbol{U}}_{\boldsymbol{r}}\right)=\mathbf{Z}_{\mathbf{r}} \tilde{\boldsymbol{U}}_{\boldsymbol{r}}+\tilde{\boldsymbol{\lambda}}-\tilde{\boldsymbol{F}}_{\boldsymbol{r}}
$$

\section{Dynamic response with fretting-wear}

Wear is a very slow phenomenon and the depth of wear is very small compared with the dimension of the structures in contact. We can neglect the modifications of the mass and stiffness matrices due to wear. Moreover in this paper we will neglect the modifications of the coefficients of friction and hardness due to wear. Wear appears here as a permanent normal displacement of the interface at the scale of one period in the response of the system. Therefore, the main idea is to split time into two different scales, indeed we consider that wear doesn't change the aspect of the periodic response on a little lapse of time. On this period it's still possible to describe displacements and contact forces with Fourier series. On a long duration Fourier coefficients will evolve as functions of a time variable linked with the fretting-wear phenomenon. This procedure belongs to the family of multiscale methods, described by Nayfeh and Mook $^{21}$ and by Meirovitch ${ }^{22}$ for a single harmonic balance. Cusumano and Chatterjee ${ }^{23}$ use a time scale separation with an averaging procedure to estimate qualitatively the dynamics of damage evolution. Here we will also use time scales splitting with an averaging procedure on each period. The Fourier series of (9) becomes:

$$
\boldsymbol{U}(\tau, \eta)=\tilde{\boldsymbol{U}}_{0}(\eta)+\sum_{n=1}^{N h} \tilde{\boldsymbol{U}}_{n, c}(\eta) \cos (n \tau)+\tilde{\boldsymbol{U}}_{n, s}(\eta) \sin (n \tau)
$$

where $\tau$ is the fast time scale linked with the periodic response and $\eta$ the slow time scale linked with fretting-wear phenomena. It's possible to define $\eta$ as a multiple of the period $T$ of fretting-wear cycles. Fretting-wear doesn't change the pulsation of the response because 
it is ruled by the pulsation of the excitation, which isn't affected by fretting-wear. Wear depth doesn't depend on $\tau$ because it doesn't evolve on a single period, that's why wear depends only on $\eta$.

$$
W^{M}=W^{M}(\tau, \eta)=W^{M}(\eta)
$$

The evolution of wear after one period is for each node in contact:

$$
\Delta W^{M}(\eta)=\int_{\text {Period }}\left|\lambda^{N}(\eta, \tau)\right| \cdot\left\|\dot{\boldsymbol{U}}_{\boldsymbol{T}}(\eta, \tau)\right\| d \tau
$$

The equation of motion (11) in the frequency domain is a little bit changed:

$$
\mathbf{Z}_{\mathbf{r}} \tilde{\boldsymbol{U}}_{\boldsymbol{r}}(\eta)+\tilde{\boldsymbol{\lambda}}(\eta)=\tilde{\boldsymbol{F}}_{\boldsymbol{r}}
$$

The term concerning wear will appear in the augmented Lagrangian used to solve this equation with the constrains of contact with friction. This procedure is described in the next part.

\section{Calculation of the contact forces}

Solving (12) requires to know the expression of the contact forces in the frequency domain. Unfortunately it's not possible to calculate a priori the multi-harmonic vector $\tilde{\boldsymbol{\lambda}}$ of Lagrange multipliers in the Galerkin procedure, indeed the function is very non-smooth. To undertake this difficulty it is common to use the Alternating Frequency Time method (AFT). Cameron and Griffin ${ }^{24}$ pioneered the development of the AFT. Displacements and velocities are calculated in the frequency domain and transformed into the time domain using an inverse FFT procedure (iFFT). In the time domain a non-linear relation gives the contact forces, which are transformed back into the frequency domain using a FFT procedure. In the time domain, contact forces may be evaluated through different methods. The easiest one is to regularize the sign function, depending on the velocity in the evaluation of Coulomb's forces, by another function which is continuous. It allows a direct computation of the nonlinear friction forces ${ }^{25,26}$. The use of a penalty method is another popular method ${ }^{14,26-29}$. This penalty softens the contact between interfaces in the tangential direction (Coulomb's friction) and in the normal direction (unilateral constrained contact conditions). The additional stiffnesses may represent a damper stiffness or the contact asperities stiffness. In case of the FEM they translate the microslip which occurs in micro-scale to the macro-scale of 
finite elements. Moreover these stiffnesses may be linked to material properties, which are modified due to fretting-wear of interfaces. These contact penalty strategies require an interactive computation of the friction forces in the time domain where the separation/stick/slip transitions have to be determined at each step.

Another method has been proposed by Nacivet et $a .^{2}$ and uses augmented Lagrangians which allow to calculate without any softening of the non-smooth contact law concerning the friction forces. A time-marching procedure in the time domain is also required. This Dynamic Lagrangian Frequency-Time method (DLFT) has been successfully used to predict friction damping in blade attachments ${ }^{1}$ and to quantitfy the efficiency of friction ring dampers ${ }^{27}$. In this method, the penalty term is the same as in the augmented Lagrangian and the final (converged) result does not depend on this term, but the stability of the procedure depends on this term. Good results are generally obtaind when it is taken equal to the spectral radius of the dynamic stiffness matrix.

In the next equations, the term $\eta$ is omitted in the expression of Fourier coefficients. In the frequency domain, the Lagrange multiplier $\boldsymbol{\lambda}$ is formulated as a penalization of the equations of motion on the tangential and normal directions:

$$
\begin{aligned}
& \tilde{\boldsymbol{\lambda}}^{\boldsymbol{T}}=\tilde{\boldsymbol{F}}_{\boldsymbol{r}}^{T}-\mathbf{Z}_{\mathbf{r}} \tilde{\boldsymbol{U}}_{\boldsymbol{r}}+\epsilon_{T}\left(\tilde{\boldsymbol{U}}_{\boldsymbol{r}}^{T}-\tilde{\boldsymbol{X}}_{\boldsymbol{r}}^{T}\right), \\
& \tilde{\boldsymbol{\lambda}}^{N}=\tilde{\boldsymbol{F}}_{\boldsymbol{r}}^{N}-\mathbf{Z}_{\mathbf{r}} \tilde{\boldsymbol{U}}_{\boldsymbol{r}}+\epsilon_{N}\left(\tilde{\boldsymbol{U}}_{\boldsymbol{r}}^{N}-\boldsymbol{W}-\tilde{\boldsymbol{X}}_{\boldsymbol{r}}^{N}\right),
\end{aligned}
$$

$\epsilon_{T}$ and $\epsilon_{N}$ are penalty coefficients, $\tilde{\boldsymbol{X}}_{\boldsymbol{r}}$ is a new vector of relative displacements, which is computed in the time domain. It will be seen that it corresponds to the relative displacements which fulfill the contact and friction law in the time domain. The pair $\left(\tilde{\boldsymbol{\lambda}}, \tilde{\boldsymbol{X}}_{\boldsymbol{r}}\right)$ is determinated through an $A F T$ procedure. Equation (12) reduces to $f\left(\tilde{\boldsymbol{U}}_{\boldsymbol{r}}\right)=\epsilon\left(\tilde{\boldsymbol{U}}_{\boldsymbol{r}}-\boldsymbol{W}-\tilde{\boldsymbol{X}}_{\boldsymbol{r}}\right)$. The convergence ensures that the time domain $\tilde{\boldsymbol{X}}_{\boldsymbol{r}}$ and frequency domain $\tilde{\boldsymbol{U}}_{\boldsymbol{r}}$ match with respect of wear condition.

\section{E. Prediction/correction in the time domain}

Now let us present the prediction/correction procedure in the time domain summarized in figure 2. The contact forces are calculated in time domain where the transition criteria 


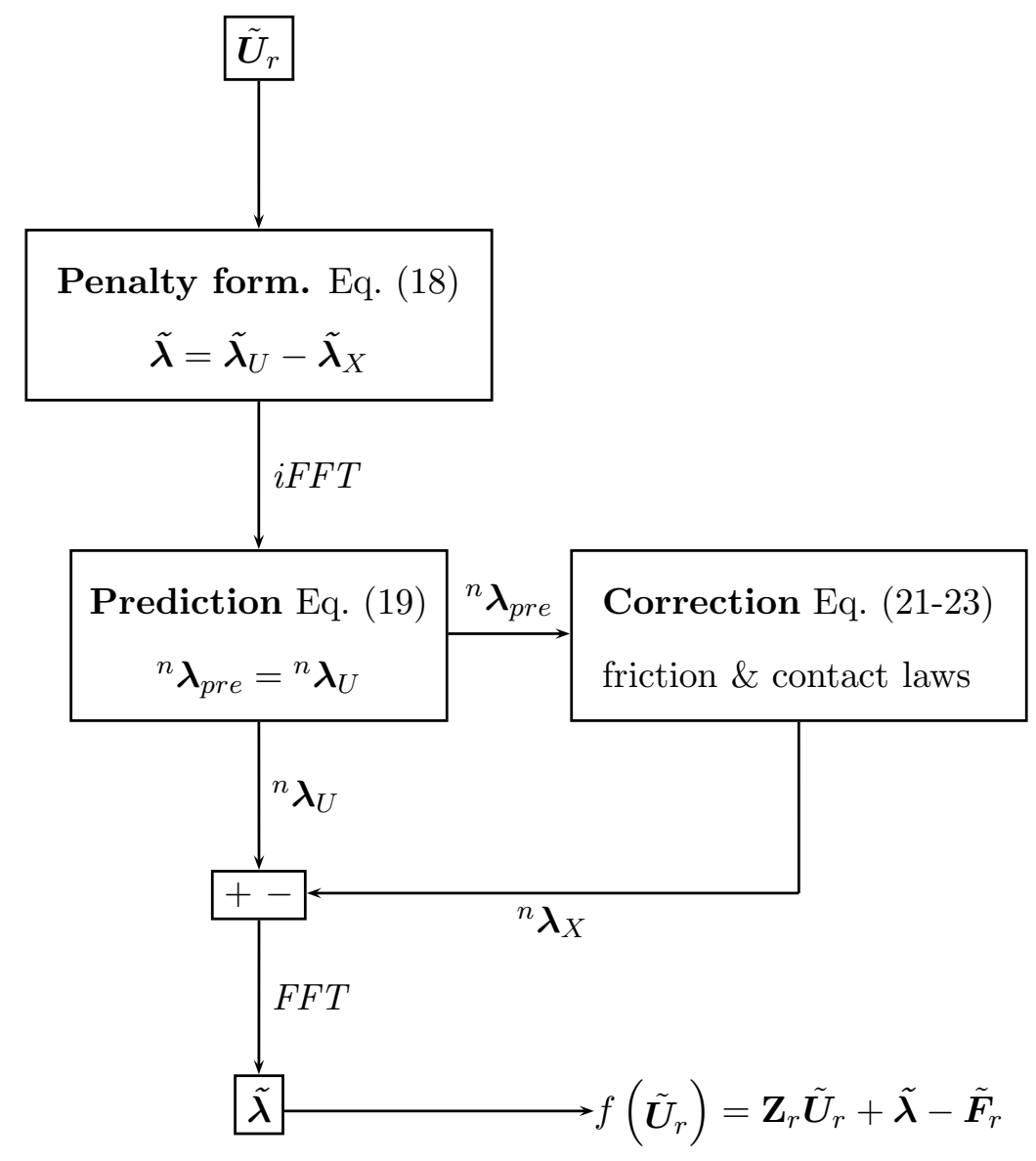

Figure 2: Computation of the Lagrangian vector with wear

between the three possible states are easily formulated. Equation (17) is reformulated as:

$$
\tilde{\boldsymbol{\lambda}}=\left(\begin{array}{c}
\tilde{\boldsymbol{\lambda}}^{T} \\
\tilde{\boldsymbol{\lambda}}^{N}
\end{array}\right)=\underbrace{\tilde{\boldsymbol{F}}_{\boldsymbol{r}}-Z_{r} \tilde{\boldsymbol{U}}_{\boldsymbol{r}}+\epsilon\left(\tilde{\boldsymbol{U}}_{\boldsymbol{r}}-\boldsymbol{W}\right)}_{\tilde{\boldsymbol{\lambda}_{u}}}-\underbrace{\epsilon \tilde{\boldsymbol{X}}_{\boldsymbol{r}}}_{\tilde{\boldsymbol{\lambda}_{x}}},
$$

The period is split into $N$ time steps. In this time domain each multi-harmonic vector has a counterpart. $\tilde{\boldsymbol{\lambda}}, \tilde{\boldsymbol{\lambda}}_{u}$ and $\tilde{\boldsymbol{\lambda}}_{x}$ have respectively $\left\{\boldsymbol{\lambda}^{n}\right\}_{n=1 . . N},\left\{\boldsymbol{\lambda}_{u}^{n}\right\}_{n=1 . . N}$ and $\left\{\boldsymbol{\lambda}_{x}^{n}\right\}_{n=1 . . N}$ as time domain equivalents. These vectors are obtained from frequency domain variables through an iFFT procedure. A prediction/correction is used to compute the contact forces. At each time increment it assumes that the contact node is in stick situation, thus the node doesn't move and $\lambda_{x}^{n, T}=\lambda_{x}^{n-1, T}$ and $\lambda_{x}^{n, N}=0$. The predicted contact forces are:

$$
\lambda_{p r e}^{n, T}=\lambda_{u}^{n, T}-\lambda_{x}^{n-1, T}, \quad \lambda_{p r e}^{n, N}=\lambda_{u}^{n, N}
$$


The corrected contact forces will be:

$$
\lambda^{n}=\lambda_{u}^{n}-\lambda_{x}^{n}
$$

$\lambda_{x}^{n}$ will we be calculated to satisfy the contact and friction laws.

1. Separation: $\lambda_{\text {pre }}^{n, N} \geq 0$, the contact is lost and the forces should be zero

$$
\lambda_{x}^{n}=\lambda_{u}^{n}
$$

2. Stick: $\lambda_{\text {pre }}^{n, N}<0$ and $\left\|\lambda_{\text {pre }}^{n, T}\right\|<\mu\left|\lambda_{\text {pre }}^{n, N}\right|$

In this case, the prediction verifies the contact conditions:

$$
\lambda_{x}^{n, N}=0, \quad \lambda_{x}^{n, T}=\lambda_{x}^{n-1, T},
$$

3. Slip: $\lambda_{\text {pre }}^{n, N}<0$ and $\left\|\boldsymbol{\lambda}_{\text {pre }}^{n, T}\right\| \geq \mu\left|\lambda_{\text {pre }}^{n, N}\right|$

Again,there is no normal relative displacement. The correction is made assuming that the tangential contact force has the same direction as the tangential predicted force. The definition of relative velocity and the respect of the Coulomb's law give:

$$
\left.\lambda_{x}^{n, N}=0, \quad \lambda_{x}^{n, T}=\lambda_{x}^{n-1, T}+\lambda_{\text {pre }}^{n, T} \quad 1-\mu \frac{\left|\lambda_{\text {pre }}^{n, N}\right|}{\left\|\lambda_{\text {pre }}^{n, T}\right\|}\right),
$$

The final step consists of transforming back the time domain updated Lagrangian in the frequency domain using FFT algorithm.

\section{F. Calculation of the wear depth}

As soon as the non-linear solver has converged, we obtain the multiharmonic vectors of displacements and contact forces and also their corrected equivalents in the time domain. These values allow us to calculate the wear rate for one period using equation (15). The easiest strategy to compute wear evolution is to calculate wear after each period and to update vector $\boldsymbol{W}$ for the next period.

$$
W^{M}\left(\eta^{k+1}\right)=W^{M}\left(\eta^{k}\right)+\Delta W^{M}\left(\eta^{k+1}\right),
$$

Even though HBM/AFT is a fast method compared with temporal integration, the calculation of frequency responses for industrial applications can last hours (even without 


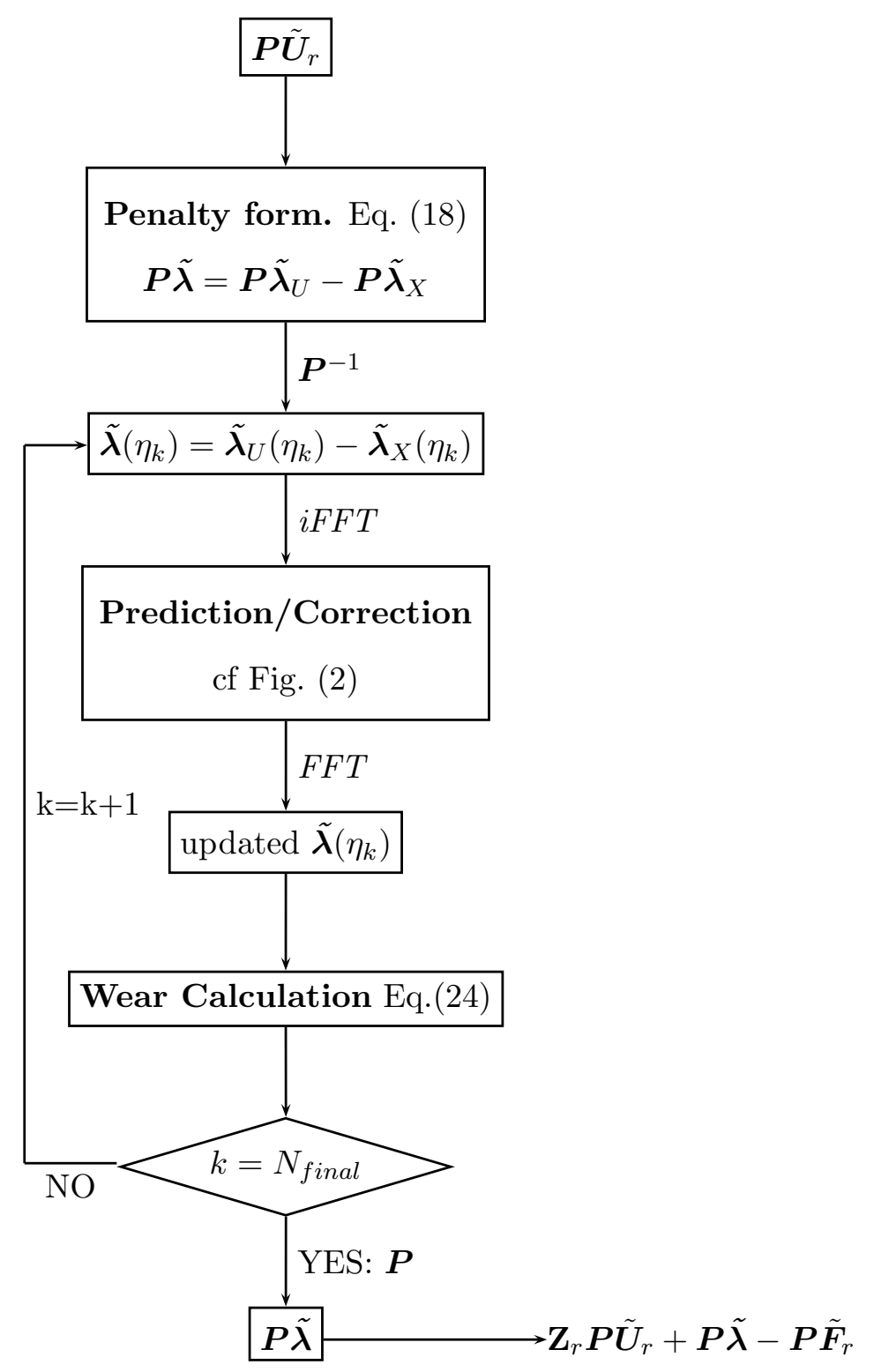

Figure 3: Computation of the Lagrangian vector

fretting-wear). Therefore, calculating wear evolution with a direct time integration method will be very long. Presently, this method is used to understand the coupling phenomena between dynamics and fretting-wear. But another method is proposed to calculate the global response on $\tau$ and $\eta$. The idea is close to the principle of HBM, indeed we use a basis of Fourier series functions to represent the time evolution in $\tau$, which allows to transform the non-linear equations in an algebraic problem. The same idea is applied with $\eta$ but it gives 
a basis of monomial series. Thus all the Fourier coefficients and wear are defined as

$$
\begin{aligned}
W(\eta) & =W_{0}+W_{1} \eta+W_{2} \eta^{2}+\cdot \\
\tilde{\lambda}(\eta) & =\tilde{\lambda}_{0}+\tilde{\lambda}_{1} \eta+\tilde{\lambda}_{2} \eta^{2}+\cdots \\
\tilde{U}(\eta) & =\tilde{U}_{0}+\tilde{U}_{1} \eta+\tilde{U}_{2} \eta^{2}+\cdots
\end{aligned}
$$

Let us introduce operator $\boldsymbol{P}$ and its inverse $\boldsymbol{P}^{-1}$, such as $\boldsymbol{P} \tilde{\boldsymbol{U}}_{r}$ is the vector of the multiharmonic polynomial coefficients. Its size is $\left(2 * N_{h}+1\right) \cdot(m+1)$, where $m$ designates the degree of the polynomial.

$$
\boldsymbol{P} \tilde{\boldsymbol{U}}_{\boldsymbol{r}}=\left[\tilde{\boldsymbol{U}}_{\mathbf{o}, \mathbf{o}}, \cdots, \tilde{\boldsymbol{U}}_{\mathbf{o}, \boldsymbol{m}}, \tilde{\boldsymbol{U}}_{1,0, c}, \tilde{\boldsymbol{U}}_{1,0, s}, \cdots, \tilde{\boldsymbol{U}}_{N_{h}, m, s}\right]^{T}
$$

The first subscript is the harmonic order and the second subscript corresponds to the degree of the monomial in the polynomial. Equation (16) is transformed as:

$$
\mathrm{Z}_{\mathrm{r}}^{\mathrm{p}} \boldsymbol{P} \tilde{U}_{\boldsymbol{r}}+\boldsymbol{P} \tilde{\lambda}=\boldsymbol{P} \tilde{\boldsymbol{F}}_{\boldsymbol{r}}
$$

The procedure of prediction/correction to calculate contact force and wear is conserved, but there are new steps. Indeed, now two time scales are considered. The procedure is illustrated in figure 3. Thus, the predicted forces are predicted from equation(29) the same way as in the precedent part, but now we have a double discretisation in the time domain: one for each period and one for long times, both are linked with $\eta$. Each time step is defined by a pair $\left(t^{n}, \eta^{k}\right)$. To transform the variable of equation (29) a calculation of the multiharmonic coefficient function must first be performed at each $\eta^{k}$ and, then, an iFFT procedure must be used. At each time step $\left(t^{n}, \eta^{k}\right)$ contact forces are corrected to satisfy the contact and friction conditions. At the end of period $k$ wear is updated for the next period. At the end of the loop on $(n, k)=\left(N, N_{\text {final }}\right)$, we have obtained the dynamical evolution on each period (time scale: $\tau$ ), the evolution of contact forces due to wear (time scale: $\eta$ ) and wear at each contact node. This vectors are transformed in the frequency domain using FFT and the coefficients of polynomial from the value of multiharmonic coefficient at each $\eta^{k}$ using $\boldsymbol{P}$ :. The new values are introduced in equation (29) to verify the convergence.

\section{NUMERICAL EXAMPLE}

The method developed above is applied to a simple model with two degrees of freedom which allows one to understand the coupling between wear and dynamics. 


\section{A. 2-DOF with wear}

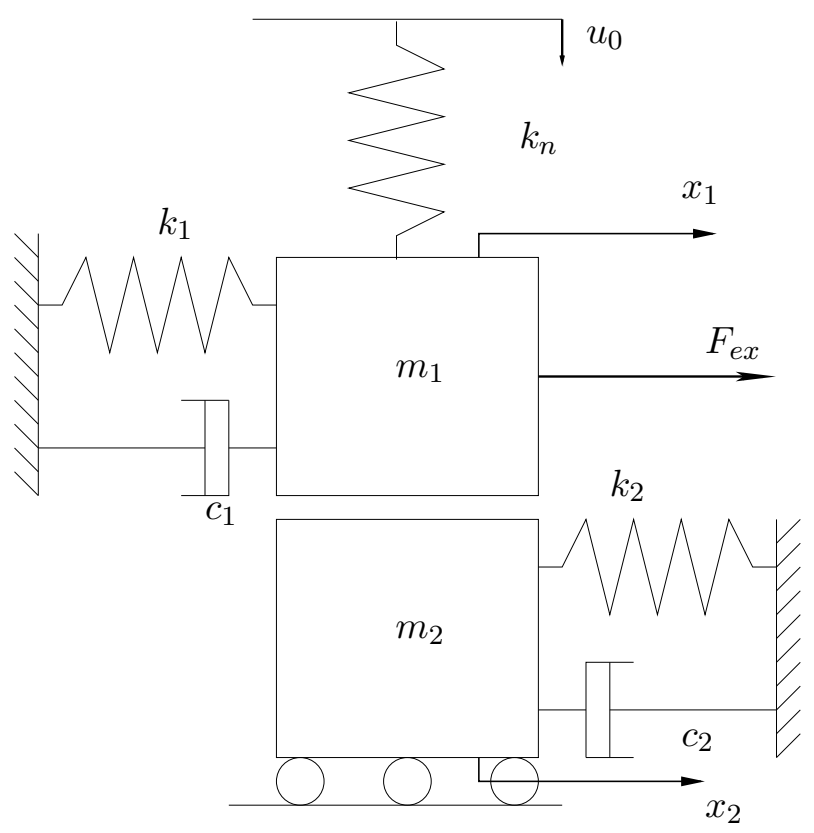

Figure 4: 2-DOF model

To start with, let us study a system made of two masses in contact with friction. The movements are only in the tangential direction. On the normal direction is applied a displacement which constrains a spring. The system is represented in figure 4 . The non-linear dynamics equations are only on the tangential direction. On the normal direction, only the first mass will wear and the wear depth leads to the relaxation of the spring and the normal contact force decreases. The equations of this system are:

$$
\begin{aligned}
& m_{1} \ddot{x_{1}}+c_{1} \dot{x_{1}}+k_{1} x_{1}+F_{n l}=F_{e x}, \\
& m_{2} \ddot{x_{2}}+c_{2} \dot{x_{2}}+k_{2} x_{2}-F_{n l}=0, \\
& \Delta w(n T)=K_{w} \int_{n T}^{(n+1) T} F_{N}\left\|\dot{x_{2}}-\dot{x_{1}}\right\| d t, \\
& F_{N}=k_{n} \cdot\left(u_{0}-w\right),
\end{aligned}
$$

$F_{n l}$ designates the tangential friction forces which must respect Coulomb's law. To solve the non-linear problem, the DLFT is used with different numbers of harmonics to test the influence of this parameter. The choice of relaxing the normal force when wear increases 
is due to the fact that in a real 3D problem the whole contact area is not in situation of stick-slip, that is why areas will remain without wear (total sticking zone). The result is the decrease of normal pressure in the area of wear. In this system, unit masses have been chosen, $k_{1}=\pi^{2}, k_{2}=7 \pi^{2}$ and the viscous damping ratio is $0.1 \%$. Thus, the two modes (stick situation and mass $m_{1}$ free) are respectively at $1 \mathrm{~Hz}$ and $0.5 \mathrm{~Hz}$. An initial normal force of $10 N\left(k_{n}=1.10^{7} \mathrm{Nm}^{-1}, u_{0}=1.10^{-3} \mathrm{~mm}\right)$ and a friction coefficient $\mu=1$ are imposed. The coefficient of wear rate $K_{w}$ is arbitrarily chosen equal to $1.010^{-6}$. We impose an excitation force in cosine and we will study three cases for the amplitude of excitation: $6 \mathrm{~N}, 10 \mathrm{~N}, 14 \mathrm{~N}$. Indeed there are three possible situations depending on the ratio $r=\left(\frac{F_{e x}}{\mu F_{N}}\right)$ if the excitation is too small the masses $m_{1}$ and $m_{2}$ are all the time in sticking situation, if the ratio is bigger than a certain value (not calculated but depending on the dynamical properties of the system) the mass $m_{1}$ is during one period often in sliding situation, and the third case occurs if the sliding situation appears but the sticking duration is longer in one period. These three cases modify the dynamic response and the wear evolution. The first case isn't studied because wear doesn't occur.

With this model let us consider the evolution of wear as a function of the number of fretting cycle and for different frequencies. We plot the frequency response function of mass $m_{1}$ as a function of the number of fretting cycles. A test has been performed to determine the influence of the number of harmonics on the precision of the results, the reference is given by a direct time integration. 21 harmonics are finally used . The results in figure 5 and figure 6 give the calculated FRFs for different ratios of excitation force.

Figure 5 shows that for certain ratios subharmonics resonances exist. Let us consider the behaviour of the modal response of the system in the presence of wear.

\section{DLFT method and direct time integration on the slow time scale}

First we impose a level of excitation force of $6 \mathrm{~N}$. The evolution of the wear depth is calculated as a function of the frequency and of the number of fretting cycles. The results are reported in figure $7 \mathrm{a}$.

We can see what happens in figure 8 where are reported FRF of mass $m_{1}$ free, mass $m_{1}$ and $m_{2}$ in stick situation and pseudo-FRF for non-linear(NL) system at the initial cycle and after 100 normalized fretting-wear cycles. 


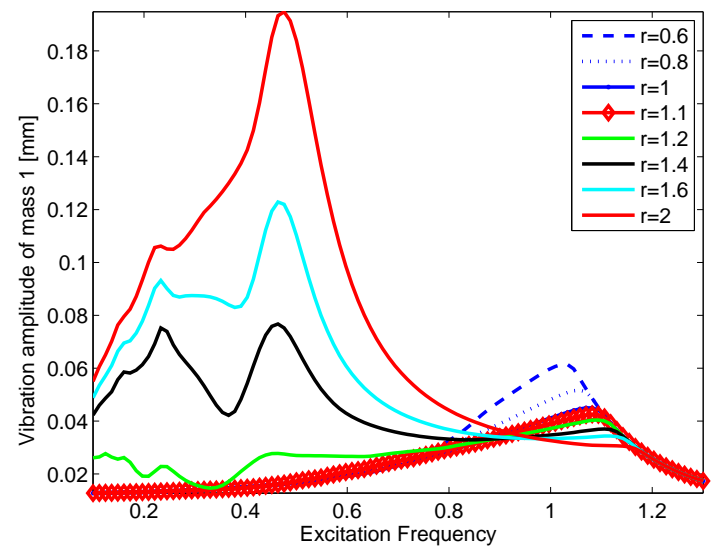

Figure 5: Frequency response of mass $m_{1}$ for several levels of excitation force

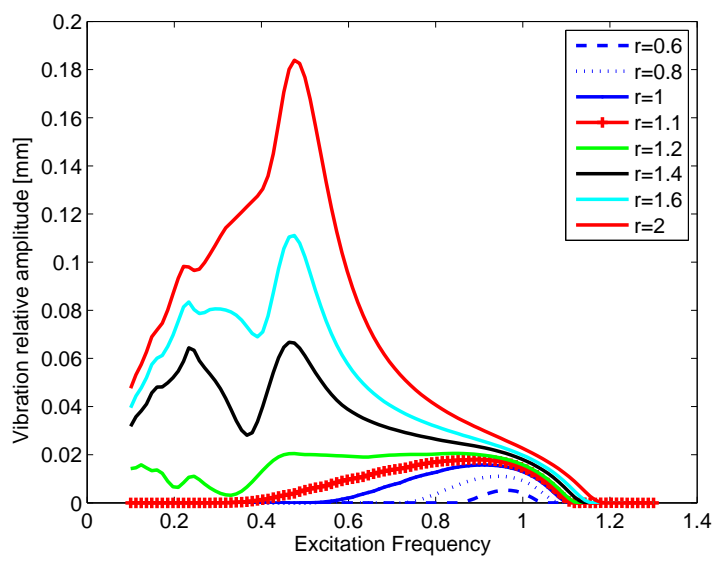

Figure 6: Frequency response of relative displacement for several levels of excitation force

They show that for this ratio of excitation wear doesn't appear in all the ranges of frequency. The plateau after a certain number of cycles exists. The functions giving the evolution of wear have points of maximal slope. We can separate evolution in three steps. First wear evolution is low because the relative speed is low. After that there is an area where the wear evolution accelerates because of the increase of relative displacement due to the decrease of the normal force and at the end of the evolution the displacement is important but the normal force is nearly zero and wear stops. The point of maximal slope corresponds to the maximum of wear rate. This figure shows the influence of dynamics on wear evolution. Figure 9a illustrates the influence of wear on the dynamic response. In this 


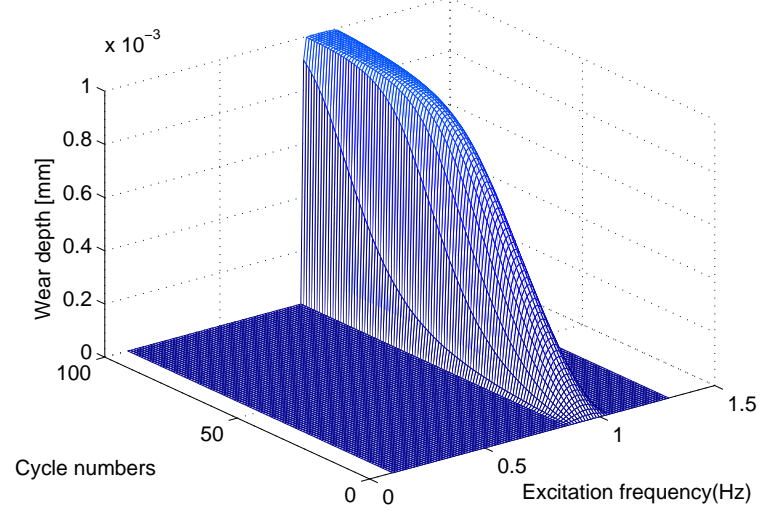

(a)

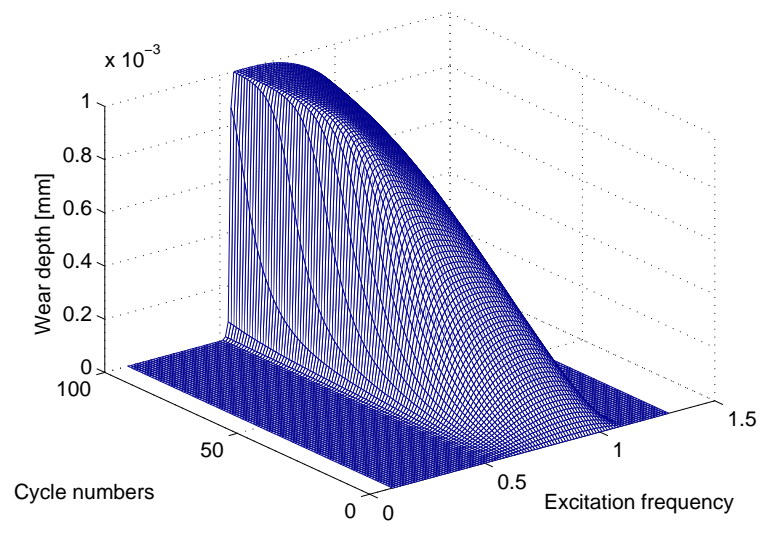

(b)

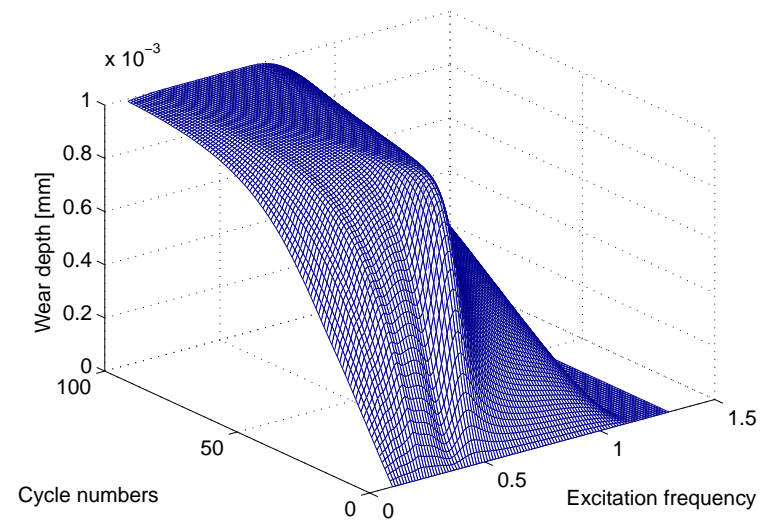

(c)

Figure 7: Wear depth evolution on a frequency range for $F_{e x}=6 N(\mathrm{a}), F_{e x}=10 N(\mathrm{~b})$ and $F_{e x}=14 N(\mathrm{c})$ 


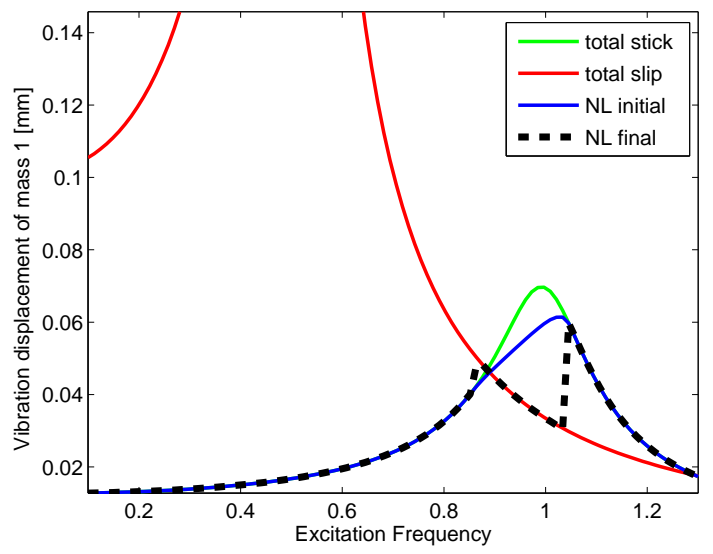

Figure 8: pseudo-Frequency response of vibration amplitude of mass 1 for 4 cases

figure two resonances appear with the increase of wear. Due to wear, the behaviour changes. With wear augmentation, mass $m_{1}$ is more and more in slip situation and the system tends to a system where mass $m 1$ is free.

The second imposed excitation force is $14 N$. Figures $9 \mathrm{c}$ and $7 \mathrm{c}$ show respectively the pseudo-FRF of mass $m_{1}$ and wear evolution. For this ratio of excitation force, the frequency slot where wear appears is more important and there is a frequency where wear evolution functions don't have point of maximal slope. In this case for initial fretting cycle, sliding period longs more than sticking period in one vibration period and wear rate is maximum in this cycle and decrease with fretting-wear cycle. For this excitation force the system is closer to sliding mode than sticking mode, that's why wear is faster at about $0.5 \mathrm{~Hz}$ where there is maximum relative displacement. Figure $9 \mathrm{~b}$ and $7 \mathrm{~b}$ represent vibration response and wear evolution for $F_{e x}=10 \cos (\omega t)$. It is an intermediate situation where the resonance without wear is at $1 \mathrm{~Hz}$ and when wear increases resonance appears at $0.5 \mathrm{~Hz}$. Theses three examples show how coupling between wear and vibration can be illustrated for a 2-DOF oscillator.

Now lets us consider the importance of the number of harmonics on the results. We have calculated for different harmonics number and drawn the wear evolution in figure 10 with $F_{\text {ex }}=10 \mathrm{~N}$ and $f=0.7 \mathrm{~Hz}$. This figure shows that the convergence increases with the harmonics number. A large number of harmonics is not necessary to have an acceptable result. Indeed for more complex structures with a lot of degrees of freedom it's interesting 


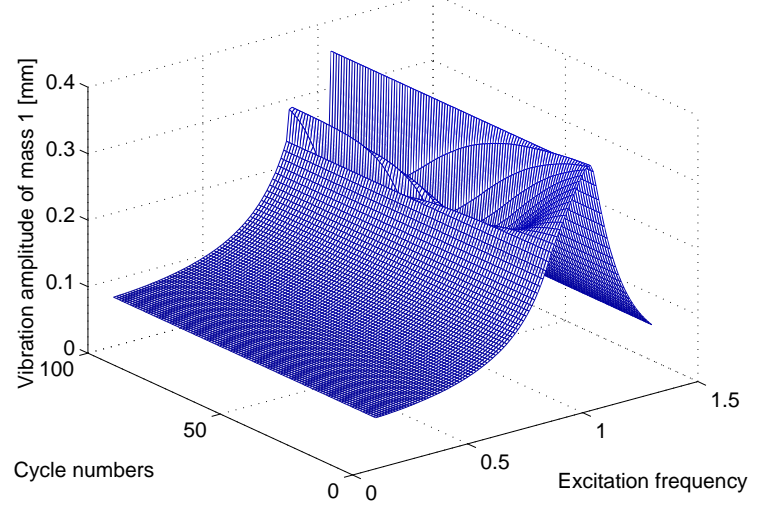

(a)

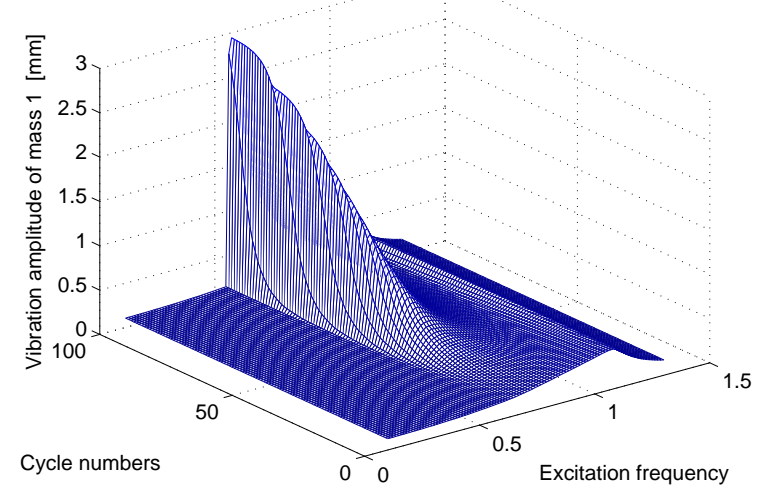

(b)

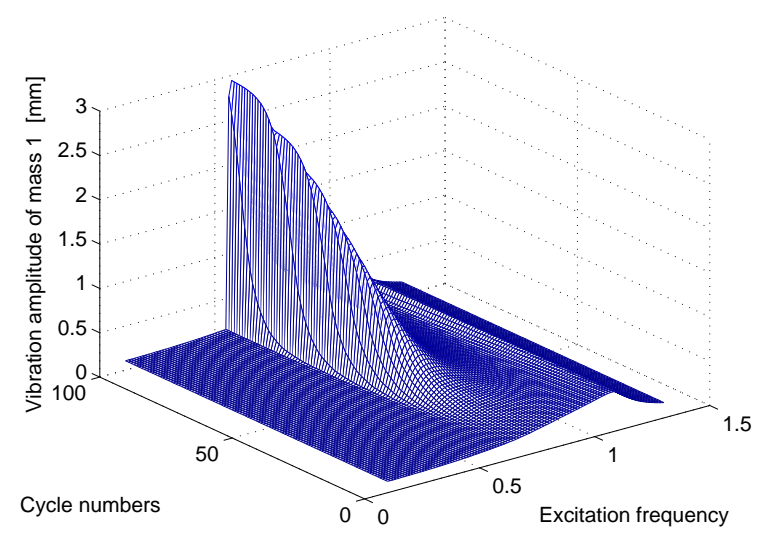

(c)

Figure 9: pesudo-Frequency Response of vibration amplitude of mass 1 for $F_{e x}=6 \mathrm{~N}$ (a), $F_{e x}=10 N(\mathrm{~b})$ and $F_{e x}=14 N(\mathrm{c})$ 


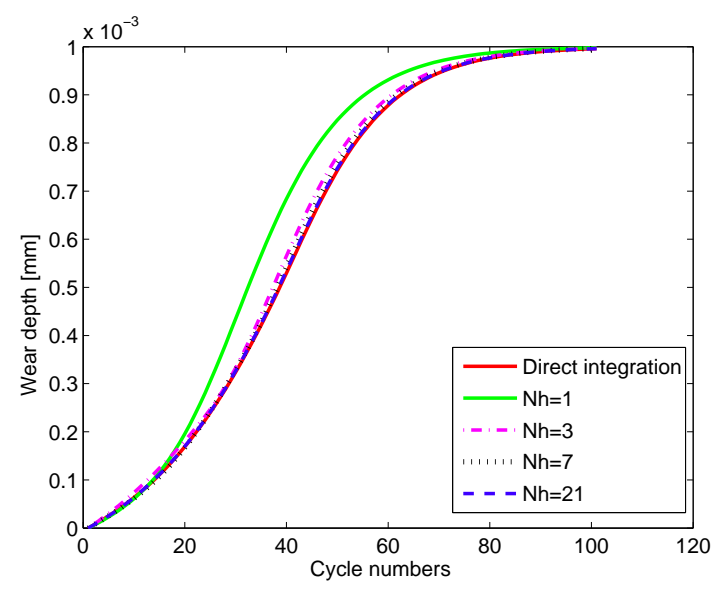

Figure 10: Wear depth evolution with different harmonics orders

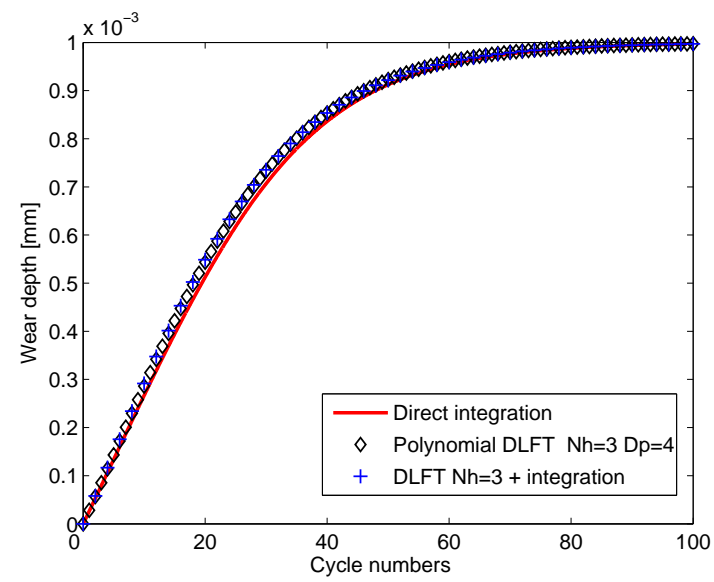

Figure 11: Wear depth evolution with three methods

not to increase harmonics number but to decrease the calculation time.

\section{Polynomial approximation of wear evolution}

Now lets us present the results obtained with the method where the evolution in $\eta$ is assumed to be polynomial. We have selected $N_{h}=3$ and a degree of polynom equal to 4 . The level of excitation force is $10 \mathrm{~N}$. The wear evolution is illustrated in figure 11 for direct time integration, for the DLFT with integration in $\eta$ and polynomial DLFT. Figure 12 shows wear evolution, results looks like results found with DLFT and for direct time integration 


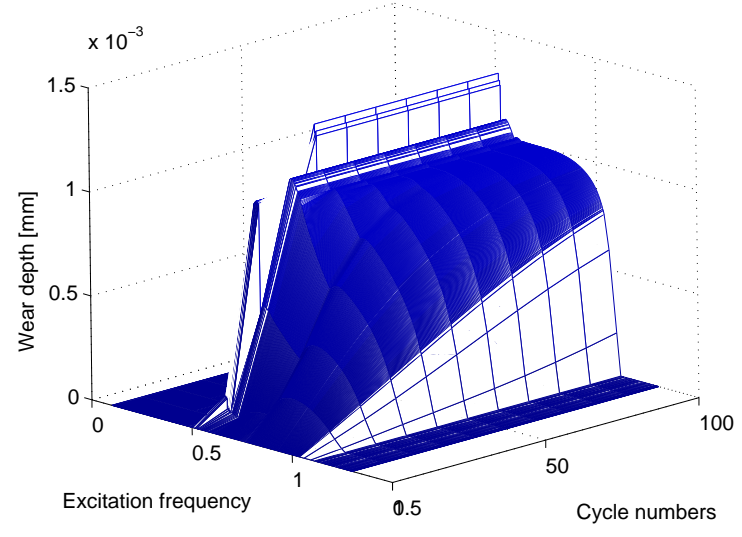

Figure 12: Wear depth evolution with polynomial approach for $F_{e x}=10 \mathrm{~N}$

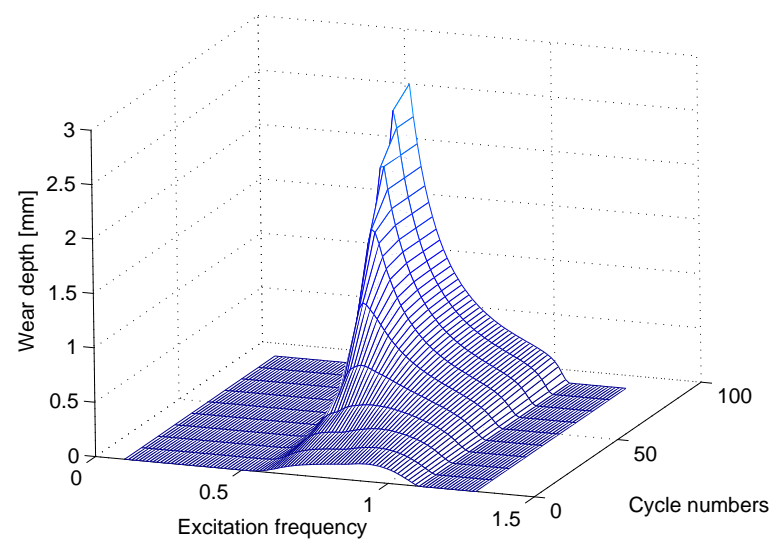

Figure 13: Relative displacements with DLFT \& integration

in $\eta$ in figure $7 \mathrm{~b}$. To compare both, the evolution of relative displacement in frequency and $\eta$ are drawn. The DLFT results are in figure 13 and the results from polynomial approach in figure 14. Our new method allow us to approximate results found with multiple DLFT with one non-linear calculation for each frequency. Nevertheless polynomial approximation isn't the most adapted function to fit wear evolution and future work will try to use other functions as Weibull function or similar functions used in maintenance engineering. 


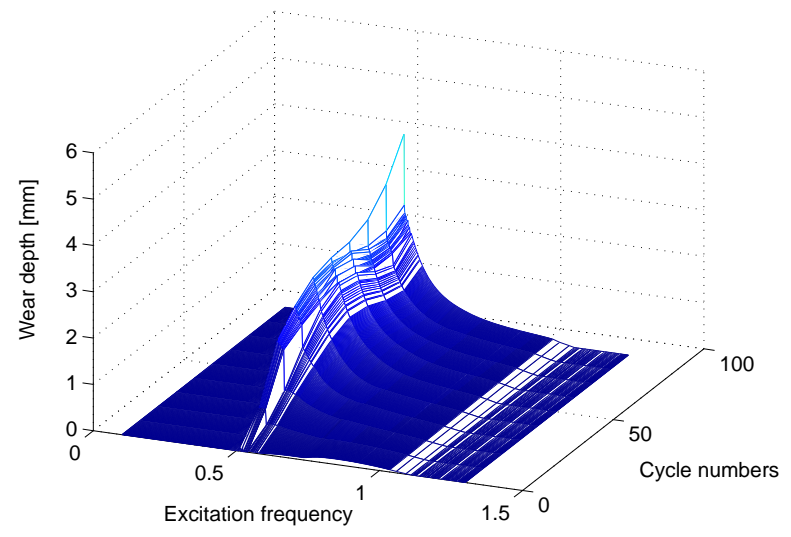

Figure 14: Relative displacements with polynomial DLFT

\section{CONCLUSIONS}

Two methods to predict wear and vibration response have been proposed. The efficiency of the first numerical method - DLFT \& time integration - is proved for simple examples. It would be slow for industrial structures but has allowed us to understand the coupling between wear and dynamics. The second method - with polynomial projection - needs more developments to be adapted for industrial structures. The future works will focus on developing new functions to formulate wear on the slow time scale, especially above the point of maximal slope.A special attention will be paid to convergence issues

\section{ACKNOWLEDGMENTS}

Thanks go to Snecma for its technical and financial support. This work takes place in the framework of the MAIA mechanical research and technology program sponsored by CNRS, ONERA and SAFRAN Group.

* Electronic address: loic.salles@ec-lyon.fr

1 D. Charleux, C. Gibert, F. Thouverez, and J. Dupeux, Numerical and Experimental Study of Friction Damping in Blade Attachments of Rotating Bladed Disks, International Journal of 
Rotating Machinery 2006 (2006).

2 S. Nacivet, C. Pierre, F. Thouverez, and L. Jezequel, A dynamic Lagrangian frequency-time method for the vibration of dry-friction-damped systems, Journal of Sound and Vibration 265, 201 (2003).

3 L. Gallego, D. Nélias, and C. Jacq, A Comprehensive Method to Predict Wear and to Define the Optimum Geometry of Fretting Surfaces, Journal of Tribology 128, 476 (2006).

4 C. Paulin, S. Fouvry, and S. Deyber, Wear kinetics of Ti-6Al-4V under constant and variable fretting sliding conditions, Wear 259, 292 (2005).

${ }^{5}$ G. Levy, Modeling of Coulomb Damping and Wear of Vibrating Systems Wear 64, 57 (1980).

6 W. Sextro, Dynamical Contact Problems with Friction: Models, Methods, Experiments and Applications (Springer, 2002).

7 H. Meng and K. Ludema, Wear models and predictive equations: their form and content, Wear 181, 443 (1995).

8 S. Fouvry, T. Liskiewicz, P. Kapsa, S. Hannel, and E. Sauger, An energy description of wear mechanisms and its applications to oscillating sliding contacts, Wear 255, 287 (2003).

9 J. Archard, Contact and rubbing of flat surface J. Appl. Phys 24, 981 (1953).

10 I. McColl, J. Ding, and S. Leen, Finite element simulation and experimental validation of fretting wear, Wear 256, 1114 (2004).

11 M. Öqvist, Numerical simulations of mild wear using updated geometry with different step size approaches, Wear 249, 6 (2001).

12 P. Põdra and S. Andersson, Wear simulation with the Winkler surface model, Wear 207, 79 (1997).

13 G. Sfantos and M. Aliabadi, Application of BEM and optimization technique to wear problems, International Journal of Solids and Structures 43, 3626 (2006).

14 J. Guillen and C. Pierre, An efficient, hybrid, frequency-time domain method for the dynamics of large-scale dry friction damped structural systems, Iutam Symposium on Unilateral Multibody Contacts (1999).

15 G. Csaba, Forced response analysis in time and frequency domains of a tuned bladed disk with friction dampers, Journal of Sound and Vibration 214, 395 (1998).

16 E. Petrov and D. Ewins, Analytical Formulation of Friction Interface Elements for Analysis of Nonlinear Multi-Harmonic Vibrations of Bladed Disks, Journal of Turbomachinery 125, 364 
(2003).

17 D. Laxalde, L. Salles, L. Blanc, and Thouverez (2008). Non-linear modal analysis for bladed disks with friction contactinterfaces, Paper 2008-GT-50860, International Gas Turbine Institute Turbo Expo 2008,

18 N. Stromberg, An augmented Lagrangian method for fretting problems European journal of mechanics. A. Solids 16, 573 (1997).

19 A. Klarbring, Derivation and analysis of rate boundary-value problems of frictional contact, Eur. J. Mech. A/Solids 9, 53 (1990).

20 J. Lemaître and J. Chaboche, Mechanics of solid materials Mécanique des matériaux solides English (Cambridge University Press, 1990).

21 A. Nayfeh and D. Mook, Nonlinear Oscillations (Wiley-Interscience, 1979).

22 L. Meirovitch, Methods of Analytical Dynamics (Dover Publications, 2004).

23 J. Cusumano and A. Chatterjee, Steps towards a qualitative dynamics of damage evolution, International Journal of Solids and Structures 37, 6397 (2000).

24 T. Cameron and J. Griffin, An alternating frequency/time domain method for calculating the steady- state response of nonlinear dynamic systems, ASME, Transactions, Journal of Applied Mechanics 56, 149 (1989).

25 K. Sanliturk, D. Ewins, R. Elliott, and J. Green, Friction Damper Optimization: Simulation of Rainbow Tests Journal of Engineering for Gas Turbines and Power 123, 930 (2001).

26 E. Petrov and D. Ewins, Models of friction damping with variable normal load for time-domain analysis of vibrations Proceedings of the International Conference on Noise and Vibration Engineering (ISMA), ISMA (2001).

27 D. Laxalde, F. Thouverez, J. Sinou, and J. Lombard, Qualitative analysis of forced response of blisks with friction ring dampers European Journal of Mechanics/A Solids 26, 676 (2007).

28 O. Poudou and C. Pierre, Hybrid frequency-time domain methods for the analysis of complex structural systems with dry friction damping, Proceedings of the AIAA/ASME/ASCE/AHS/ASC Structures, Structural Dynamics and Materials Conference, AIAA, New York 1, 111 (2003).

29 E. Ciğgeroğlu and H. Özgüven, Nonlinear vibration analysis of bladed disks with dry friction dampers, Journal of Sound and Vibration 295, 1028 (2006). 